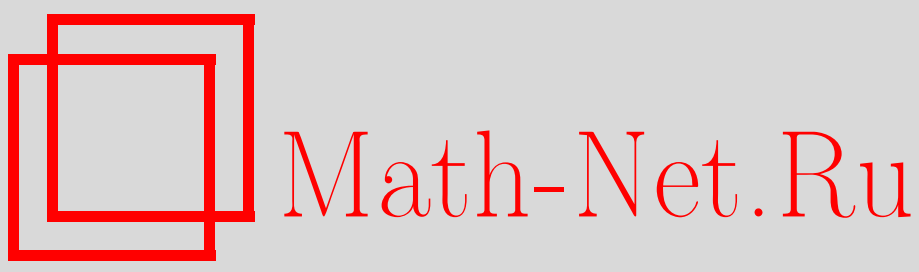

A. Silverberg, Yu. G. Zarhin, Hodge groups of abelian varieties with purely multiplicative reduction, Изв. РАН. Сер. матем., 1996, том 60, выпуск 2, 149-158

DOI: https://doi.org/10.4213/im74

Использование Общероссийского математического портала Math-Net.Ru подразумевает, что вы прочитали и согласны с пользовательским соглашением http: //www. mathnet.ru/rus/agreement

Параметры загрузки:

IP : 54.198 .187 .58

26 апреля 2023 г., 11:33:05 
УДК 513.6

\author{
A. Silverberg, Yu. G. Zarhin
}

\title{
Hodge groups of abelian varieties with purely multiplicative reduction
}

\begin{abstract}
The main result of the paper is that if $A$ is an abelian variety over a subfield $F$ of $\mathbf{C}$, and $A$ has purely multiplicative reduction at a discrete valuation of $F$, then the Hodge group of $A$ is semisimple. Further, we give necessary and sufficient conditions for the Hodge group to be semisimple. We obtain bounds on certain torsion subgroups for abelian varieties which do not have purely multiplicative reduction at a given discrete valuation, and therefore obtain bounds on torsion for abelian varieties, defined over number fields, whose Hodge groups are not semisimple.

Bibliography: 26 titles.
\end{abstract}

\section{$\S 1$. Introduction}

We show that if $A$ is an abelian variety over a subfield $F$ of $\mathbf{C}$, and $A$ has purely multiplicative reduction at a discrete valuation of $F$, then the Hodge group of $A$ is semisimple (Theorem 4.1). Since the non-semisimplicity of the Hodge group of an abelian variety can be translated into a condition on the endomorphism algebra and its action on the tangent space (see Theorem 3.1), this gives a useful criterion for determining when an abelian variety does not have purely multiplicative reduction. For abelian varieties over number fields, a result analogous to Theorem 4.1 holds where the Hodge group is replaced by a certain linear algebraic group $H_{\ell}$ over $\mathbf{Q}_{\ell}$ arising from the image of the $\ell$-adic representation associated to $A$ (see Theorem 3.2). The Mumford-Tate conjecture predicts that $H_{\ell}$ is the extension of scalars to $\mathbf{Q}_{\ell}$ of the Hodge group.

Our result generalizes a result of Mustafin (Corollary after Theorem 3.2 of [13]), which says that for a Hodge family of abelian varieties (as in [10]) admitting a "strong degeneration", generically the fibers have semisimple Hodge group. The problem of describing the Hodge group of an abelian variety with purely multiplicative reduction was posed by V. G. Drinfeld, in a conversation with Zarhin in the 1980's.

In $\S 5$ we provide bounds on torsion for abelian varieties which do not have purely multiplicative reduction at a given discrete valuation. We apply this and Theorem 4.1 to obtain bounds on torsion for abelian varieties whose Hodge groups are not semisimple.

Silverberg would like to thank MSRI and IHES for their generous hospitality, and NSF for financial support. Zarhin would like to thank the Institute für Experimentelle Mathematik for its hospitality, and Gerhard Frey for his interest in the paper and useful discussions.

\section{§ 2. Definitions, notation, and lemmas}

Suppose $A$ is an abelian variety defined over a field $F$ of characteristic zero, and $L$ is an algebraically closed field containing $F$. Write $\operatorname{End}_{F}(A)$ for the set

(C) A. Silverberg, Yu. G. Zarhin 1996 
of endomorphisms of $A$ which are defined over $F$, let $\operatorname{End}(A)=\operatorname{End}_{L}(A)$, let $\operatorname{End}^{0}(A)=\operatorname{End}(A) \otimes_{\mathbf{z}} \mathbf{Q}$, and let $\operatorname{End}_{F}^{0}(A)=\operatorname{End}_{F}(A) \otimes_{\mathbf{Z}} \mathbf{Q}$.

Suppose $K$ is a field and $\iota: K \hookrightarrow \operatorname{End}_{F}^{0}(A)$ is an embedding such that $\iota(1)=1$. Let $\operatorname{Lie}_{F}(A)$ be the tangent space of $A$ at the origin, an $F$-vector space. If $\sigma$ is an embedding of $K$ into $L$, let

$$
n_{\sigma}=\operatorname{dim}_{L}\left\{t \in \operatorname{Lie}_{L}(A): \iota(\alpha) t=\sigma(\alpha) t \text { for all } \alpha \in K\right\} .
$$

Note that $n_{\sigma}$ is independent of the choice of an algebraically closed field $L$ containing $F$. Write $\bar{\sigma}$ for the composition of $\sigma$ with the involution complex conjugation of $K$.

Definition 2.1. If $A$ is an abelian variety over an algebraically closed field $L$ of characteristic zero, $K$ is a CM-field, and $\iota: K \hookrightarrow \operatorname{End}^{0}(A)$ is an embedding such that $\iota(1)=1$, we say $(A, K, \iota)$ is of Weil type if $n_{\sigma}=n_{\bar{\sigma}}$ for all embeddings $\sigma$ of $K$ into $L$.

Lemma 2.2. If $A$ is an abelian variety defined over a field $F$ of characteristic zero, $L$ is an algebraically closed field containing $F, K$ is a CM-field, and $\iota: K \hookrightarrow$ $\operatorname{End}_{F}^{0}(A) \subseteq \operatorname{End}^{0}(A)$ is an embedding such that $\iota(1)=1$, then the following statements are equivalent:

(i) $(A, K, \iota)$ is of Weil type,

(ii) ८ makes $\mathrm{Lie}_{L}(A)$ into a free $\left(K \otimes_{\mathbf{Q}} L\right)$-module,

(iii) $\iota$ makes $\operatorname{Lie}_{F}(A)$ into a free $\left(K \otimes_{\mathbf{Q}} F\right)$-module.

Proof. Let $\Sigma$ be the set of embeddings of $K$ into $L$, let $\psi_{F}: K \otimes_{\mathbf{Q}} F \rightarrow$ $\operatorname{End}\left(\operatorname{Lie}_{F}(A)\right)$ be the homomorphism induced by $\iota$, let $\psi: K \otimes_{\mathbf{Q}} L \rightarrow \operatorname{End}\left(\operatorname{Lie}_{L}(A)\right)$ be the extension of scalars of $\psi_{F}$ to $K \otimes_{\mathbf{Q}} L$, let $m=2 \operatorname{dim}(A) /[K: \mathbf{Q}]$, let $M_{F}=\left(K \otimes_{\mathbf{Q}}\right.$ $F)^{m}$, and let $M=\left(K \otimes_{\mathbf{Q}} L\right)^{m}=M_{F} \otimes_{F} L$. Let $\psi_{F}^{\prime}: K \otimes_{\mathbf{Q}} F \rightarrow \operatorname{End}\left(M_{F}\right)$ and $\psi^{\prime}: K \otimes_{\mathbf{Q}} L \rightarrow \operatorname{End}(M)$ be the natural homomorphisms. By $\S 2.1$ of [18], for every $\sigma \in \Sigma$ we have $n_{\sigma}+n_{\bar{\sigma}}=m$. For $\alpha \in K$, taking the trace of $\psi(\alpha)$ gives

$$
\operatorname{tr}(\psi(\alpha))=\sum_{\sigma \in \Sigma} n_{\sigma} \sigma(\alpha)
$$

The traces of $\psi$ and of $\psi^{\prime}$ coincide on $K$ if and only if $n_{\sigma}=n_{\bar{\sigma}}=m / 2$ for every $\sigma \in \Sigma$. Since $K \otimes_{\mathbf{Q}} L$ is a semisimple ring, $\operatorname{Lie}_{L}(A)$ and $M$ are semisimple $\left(K \otimes_{\mathbf{Q}} L\right)$-modules. Therefore, $\operatorname{Lie}_{L}(A)$ is a free $\left(K \otimes_{\mathbf{Q}} L\right)$-module if and only if the traces of $\psi$ and of $\psi^{\prime}$ coincide on $K$. Therefore, $\operatorname{Lie}_{L}(A)$ is a free $\left(K \otimes_{\mathbf{Q}} L\right)$-module if and only if $(A, K, \iota)$ is of Weil type.

If $\operatorname{Lie}_{F}(A)$ is a free $\left(K \otimes_{\mathbf{Q}} F\right)$-module, then clearly $\operatorname{Lie}_{L}(A) \quad\left(=\operatorname{Lie}_{F}(A) \otimes_{F} L\right)$ is a free $\left(K \otimes_{\mathbf{Q}} L\right)$-module.

Conversely, if $\operatorname{Lie}_{L}(A)$ is a free $\left(K \otimes_{\mathbf{Q}} L\right)$-module, then the traces of $\psi$ and of $\psi^{\prime}$ (and therefore of $\psi_{F}$ and of $\psi_{F}^{\prime}$ ) coincide on $K$. Since $K \otimes_{\mathbf{Q}} F$ is a semisimple ring, $\operatorname{Lie}_{F}(A)$ and $M_{F}$ are semisimple $\left(K \otimes_{\mathbf{Q}} F\right)$-modules. Therefore $\operatorname{Lie}_{F}(A)$ and $M_{F}$ are isomorphic as $\left(K \otimes_{\mathbf{Q}} F\right)$-modules, i.e., $\operatorname{Lie}_{F}(A)$ is a free $\left(K \otimes_{\mathbf{Q}} F\right)$-module.

See also p. 525 of [15] for the case where $K$ is an imaginary quadratic field.

REMARK 2.3. If $A$ is an abelian variety defined over a field $F$ of characteristic zero, $K$ is a totally real number field, and $\iota: K \hookrightarrow \operatorname{End}_{F}^{0}(A)$ is an embedding such that $\iota(1)=1$, then $\iota$ makes $\operatorname{Lie}_{F}(A)$ into a free $\left(K \otimes_{\mathbf{Q}} F\right)$-module. To see this, let $L$ be an algebraically closed field containing $F$, let $\Sigma$ be the set of embeddings of 
$K$ into $L$, and let $\psi: K \rightarrow \operatorname{End}\left(\operatorname{Lie}_{L}(A)\right)$ be the homomorphism induced by $\iota$. Let $m^{\prime}=\operatorname{dim}(A) /[K: \mathbf{Q}]$. We have

$$
\operatorname{tr}(\psi(\alpha))=m^{\prime} \sum_{\sigma \in \Sigma} \sigma(\alpha)
$$

for every $\alpha \in K$, by $\S 2.1$ of [18]. Therefore, $\operatorname{Lie}_{L}(A)$ is a free $\left(K \otimes_{\mathbf{Q}} L\right)$-module. As in the proof of Lemma 2.2, it follows that $\operatorname{Lie}_{F}(A)$ is a free $\left(K \otimes_{\mathbf{Q}} F\right)$-module.

Suppose $A$ is a complex abelian variety. Let $V=H_{1}(A, \mathbf{Q})$ and let $\mathbf{S}=$ $\operatorname{Res}_{\mathbf{C} / \mathbf{R}} \mathbf{G}_{m}$. The complex structure on $A$ gives rise to a rational Hodge structure on $V$ of weight -1 , i.e., a homomorphism of algebraic groups $h: \mathbf{S} \rightarrow \operatorname{GL}(V)_{\mathbf{R}}$. Let $\mathbf{T}$ be the kernel of the norm map $\mathbf{N}: \mathbf{S} \rightarrow \mathbf{G}_{m}$. Then $\mathbf{T}(\mathbf{R})=\{x \in \mathbf{C}:|x|=1\}$.

Definition 2.4. If $A$ is an abelian variety over $\mathbf{C}$ and $V=H_{1}(A, \mathbf{Q})$, then the Hodge group $H$ is the smallest algebraic subgroup of $\mathrm{GL}(V)$ defined over $\mathbf{Q}$ such that $H(\mathbf{R})$ contains $h(\mathbf{T}(\mathbf{R}))$. Equivalently, $H$ is the largest algebraic subgroup of $\mathrm{GL}(V)$ defined over $\mathbf{Q}$ such that all Hodge classes in $V^{\otimes p} \otimes\left(V^{*}\right)^{\otimes q}$, for all non-negative integers $p$ and $q$, are tensor invariants of $H$. I.e., $H$ is the largest algebraic subgroup of GL $(V)$ defined over $\mathbf{Q}$ which fixes all Hodge classes of all powers of $A$.

It follows from the definition of $H$ that $\operatorname{End}^{0}(A)=\operatorname{End}_{H}(V)$.

If now $F$ is a number field and $\ell$ is a prime number, let $T_{\ell}(A)=\lim _{\leftarrow} A_{\ell^{r}}$ (the Tate module), let $V_{\ell}(A)=T_{\ell}(A) \otimes \mathbf{z}_{\ell} \mathbf{Q}_{\ell}$, and let $\rho_{A, \ell}$ denote the $\ell$-adic representation

$$
\rho_{A, \ell}: \operatorname{Gal}(\bar{F} / F) \rightarrow \mathrm{GL}\left(T_{\ell}(A)\right) \subseteq \mathrm{GL}\left(V_{\ell}(A)\right) .
$$

Let $G_{\ell}$ denote the algebraic envelope of the image of $\rho_{A, \ell}$, i.e., the Zariski closure in $\operatorname{GL}\left(V_{\ell}(A)\right)$ of the image of $\rho_{A, \ell}$. By [1], $G_{\ell}$ is a reductive algebraic group, and $\operatorname{End}_{F}^{0}(A) \otimes_{\mathbf{Q}} \mathbf{Q}_{\ell}=\operatorname{End}_{G_{\ell}}\left(V_{\ell}(A)\right)$. Let $H_{\ell}$ be the identity connected component of $G_{\ell} \cap \mathrm{SL}\left(V_{\ell}(A)\right)$. Then $H_{\ell}$ is a connected reductive group and $\operatorname{End}^{0}(A) \otimes_{\mathbf{Q}} \mathbf{Q}_{\ell}=$ $\operatorname{End}_{H_{\ell}}\left(V_{\ell}(A)\right)$.

We will repeatedly use the fact (see the first Theorem on p. 220 of [6]) that if $G$ is a connected linear algebraic group over a field $F$ of characteristic zero, then $G(F)$ is Zariski-dense in $G$.

Lemma 2.5. If $G$ is a reductive linear algebraic group over a field $F$ of characteristic zero, and $Z$ is the center of $G$, then $G$ is semisimple if and only if $Z(F)$ is finite.

Proof. Let $Z^{0}$ denote the identity connected component of $Z$. Since $G$ is reductive, $G$ is semisimple if and only if $Z^{0}=1$ (see the lemma on p. 125 of [6]). Since $Z^{0}(F)$ is Zariski-dense in $Z^{0}, Z^{0}=1$ if and only if $Z(F)$ is finite.

\section{$\S 3$. Semisimplicity criteria for the groups $H$ and $H_{\ell}$}

If the center of $\operatorname{End}^{0}(A)$ is a direct sum of totally real number fields, then it is well-known that the groups $H$ and $H_{\ell}$ are semisimple (see, for instance, Corollary 1 in $\S 1.3 .1$ of [24] and Lemma 1.4 of [22]). The following result follows easily from a result in [8], and characterizes the endomorphism algebras of abelian varieties whose Hodge groups are not semisimple.

Theorem 3.1. Suppose $A$ is an abelian variety defined over $\mathbf{C}$. Then the Hodge group of $A$ is not semisimple if and only if for some simple component $B$ of $A$, the center of $\operatorname{End}^{0}(B)$ is a CM-field $K$ such that $(B, K, \mathrm{id})$ is not of Weil type, with id the identity embedding of $K$ in $\operatorname{End}^{0}(B)$. 
Proof. Let $V=H_{1}(A, \mathbf{Q})$. Fix a polarization on $A$. The polarization induces a non-degenerate alternating bilinear form $\varphi: V \times V \rightarrow \mathbf{Q}$ such that $H \subseteq \operatorname{Sp}(V, \varphi)$ (see [11]). Then

$$
H(\mathbf{Q}) \subseteq \operatorname{Sp}(V, \varphi)(\mathbf{Q})=\left\{g \in \operatorname{End}(V): g g^{\prime}=1\right\},
$$

where $g \mapsto g^{\prime}$ is the involution on $\operatorname{End}(V)$ defined by

$$
\varphi(g(x), y)=\varphi\left(x, g^{\prime}(y)\right) \text { for } x, y \in V .
$$

The restriction of the involution ' to $\operatorname{End}^{0}(A)$ is the Rosati involution. Let $Z$ denote the center of $H$ and let $Z$ End denote the center of $\operatorname{End}^{0}(A)$. If $\alpha \in Z(\mathbf{Q})$, then $\alpha$ commutes with all elements of $H(\mathbf{Q})$, so $\alpha \in \operatorname{End}^{0}(A)$. Further, since $\alpha \in H(\mathbf{Q}), \alpha$ commutes with all elements of $\operatorname{End}^{0}(A)$, and therefore $\alpha \in Z_{\text {End }}$. Therefore,

$$
Z(\mathbf{Q}) \subseteq\left\{\alpha \in Z_{\text {End }}: \alpha \alpha^{\prime}=1\right\}
$$

If $A$ is isogenous to a product of two abelian varieties, then the Hodge group $H$ of $A$ is a subgroup of the product of the Hodge groups $H_{1}$ and $H_{2}$ of the factors, in such a way that for $i=1$ and 2 the restriction to $H$ of the projection map from $H_{1} \times H_{2}$ onto $H_{i}$ induces a surjective homomorphism from $H$ onto $H_{i}$ (see Proposition 1.6 of [5]). It follows easily that $H$ is semisimple if and only if both $H_{1}$ and $H_{2}$ are semisimple. We may therefore reduce to the case where $A$ is a simple abelian variety. Then the center $Z_{\text {End }}$ of $\operatorname{End}^{0}(A)$ is either a totally real number field or a CM-field.

Suppose $Z_{\text {End }}$ is totally real. Then all Rosati involutions are the identity when restricted to $Z_{\text {End }}$. By (1), $Z(\mathbf{Q}) \subseteq\{ \pm 1\}$. Therefore, $Z(\mathbf{Q})$ is finite, so $H$ is semisimple by Lemma 2.5 .

Suppose $Z_{\text {End }}$ is a CM-field $K$. Then every Rosati involution induces complex conjugation on $K$. Choose $\alpha \in K^{\times}$such that $\bar{\alpha}=-\alpha$. Then there exists a unique $K$-Hermitian form $\psi: V \times V \rightarrow K$ such that $\varphi(x, y)=\operatorname{Tr}_{K / \mathbf{Q}}(\alpha \psi(x, y))$ (see [18]). The unitary group $\mathrm{U}(V, \psi)$ is an algebraic group over $K_{0}$, the maximal totally real subfield of $K$. Let $U=\operatorname{Res}_{K_{0} / \mathbf{Q}} \mathrm{U}(V, \psi)$, let $\mathrm{SU}$ denote the kernel of the determinant homomorphism $\operatorname{det}_{K}: \mathrm{U} \rightarrow \operatorname{Res}_{K / \mathbf{Q}} \mathbf{G}_{m}$, and let $\operatorname{End}_{K}(V)$ denote the ring of $K$-linear endomorphisms of $V$. Then

$$
\mathrm{U}(\mathbf{Q})=\left\{g \in \operatorname{End}_{K}(V): \psi(g(x), g(y))=\psi(x, y) \text { for all } x, y \in V\right\}
$$

and $H \subseteq \mathrm{U} \subseteq \operatorname{Sp}(V, \varphi)$. By Lemma 2.8 of [8], $H \subseteq \mathrm{SU}$ if and only if $(A, K$,id) is of Weil type. If $H$ is semisimple, then all homomorphisms from $H$ to commutative groups are trivial. Therefore $\operatorname{det}_{K}(H)=1$, so $H \subseteq \mathrm{SU}$. Conversely, if $H \subseteq \mathrm{SU}$, then $Z(\mathbf{Q}) \subseteq \mathrm{SU}(\mathbf{Q}) \cap K$, the group of $(\operatorname{dim}(V) /[K: \mathbf{Q}])$-th roots of unity in $K$. Therefore $Z(\mathbf{Q})$ is finite and $H$ is semisimple.

Theorem 3.2. Suppose $A$ is an abelian variety defined over a number field $F$. Then the following are equivalent:

(i) $H$ is semisimple,

(ii) $H_{\ell}$ is semisimple, for one prime $\ell$,

(iii) $H_{\ell}$ is semisimple, for every prime $\ell$. 
Proof. Let $\ell$ be a prime number and let $V_{\ell}=V_{\ell}(A)$. By Theorem 3.1, it suffices to show that $H_{\ell}$ is not semisimple if and only if for some simple component $B$ of $A$, the center of $\operatorname{End}^{0}(B)$ is a CM-field $K$ such that $(B, K$,id) is not of Weil type, with id the identity embedding of $K$ in $\operatorname{End}^{0}(B)$. Since $H_{\ell}$ is connected, it is invariant under finite extensions of the number field $F$. By replacing $F$ by a finite extension, we may suppose that $\operatorname{End}^{0}(A)=\operatorname{End}_{F}^{0}(A)$.

We parallel the proof of Theorem 3.1. Fix a polarization on $A$ defined over $F$. Let $V$ and $\varphi$ be as in the proof of Theorem 3.1. Then $V_{\ell}=V \otimes_{\mathbf{Q}} \mathbf{Q}_{\ell}$. Let $\varphi_{\ell}: V_{\ell} \times V_{\ell} \rightarrow \mathbf{Q}_{\ell}$ be the $\mathbf{Q}_{\ell}$-linear extension of $\varphi$. It follows immediately from p. 516 of [25] and the definition of $H_{\ell}$ that $H_{\ell} \subseteq \operatorname{Sp}\left(V_{\ell}, \varphi_{\ell}\right)$. Let $Z_{\ell}$ denote the center of $H_{\ell}$, let $Z_{\text {End }}$ denote the center of $\operatorname{End}^{0}(A)$, and let ' denote the involution on $\operatorname{End}\left(V_{\ell}\right)$ induced by $\varphi_{\ell}$. Following the proof of Theorem 3.1, we conclude that

$$
Z_{\ell}\left(\mathbf{Q}_{\ell}\right) \subseteq\left\{\alpha \in Z_{\text {End }} \otimes_{\mathbf{Q}} \mathbf{Q}_{\ell}: \alpha \alpha^{\prime}=1\right\}
$$

If $A$ is $F$-isogenous to a product of two abelian varieties, then $H_{\ell}$ is a subgroup of the product of the corresponding groups $H_{1, \ell}$ and $H_{2, \ell}$ for the factors, in such a way that for $i=1$ and 2 the restriction to $H_{\ell}$ of the projection map from $H_{1, \ell} \times H_{2, \ell}$ onto $H_{i, \ell}$ induces a surjective homomorphism from $H_{\ell}$ onto $H_{i, \ell}$. It follows that we may reduce to the case where $A$ is $F$-simple.

If $Z_{\text {End }}$ is totally real, we conclude that $H_{\ell}$ is semisimple as in the proof of Theorem 3.1. Suppose $Z_{\text {End }}$ is a CM-field $K$ and let $K_{\ell}=K \otimes_{\mathbf{Q}} \mathbf{Q}_{\ell}$. Let $\psi$ and $U$ be as in the proof of Theorem 3.1, let $\psi_{\ell}: V_{\ell} \times V_{\ell} \rightarrow K_{\ell}$ denote the $K_{\ell}$-Hermitian form which extends the pairing $\psi$, let $\mathrm{U}_{\ell}=\mathrm{U} \times \mathbf{Q}_{\ell}$, let $\mathrm{SU}_{\ell}$ denote the kernel of the determinant homomorphism $\operatorname{det}_{K_{\ell}}: \mathrm{U}_{\ell} \rightarrow \operatorname{Res}_{K / \mathbf{Q}} \mathbf{G}_{m} \times \mathbf{Q}_{\ell}$, and let $\operatorname{End}_{K_{\ell}}\left(V_{\ell}\right)$ denote the ring of $K_{\ell}$-linear endomorphisms of $V_{\ell}$. Then

$$
\mathrm{U}_{\ell}\left(\mathbf{Q}_{\ell}\right)=\left\{g \in \operatorname{End}_{K_{\ell}}\left(V_{\ell}\right): \psi_{\ell}(g(x), g(y))=\psi_{\ell}(x, y) \text { for all } x, y \in V_{\ell}\right\}
$$

and $H_{\ell} \subseteq \mathrm{U}_{\ell} \subseteq \mathrm{Sp}\left(V_{\ell}, \varphi_{\ell}\right)$. By Lemma 2.8 of [8], $H_{\ell} \subseteq \mathrm{SU}_{\ell}$ if and only if $\left(A, K\right.$,id) is of Weil type. The group $\mathrm{SU}_{\ell}\left(\mathbf{Q}_{\ell}\right) \cap K_{\ell}$ is the finite group of $\left(\operatorname{dim}_{\mathbf{Q}_{\ell}}\left(V_{\ell}\right) / \operatorname{dim}_{\mathbf{Q}_{\ell}}\left(K_{\ell}\right)\right)$-th roots of unity in the ring $K_{\ell}$. Paralleling the proof of Theorem 3.1, $H_{\ell} \subseteq \mathrm{SU}_{\ell}$ if and only if $H_{\ell}$ is semisimple.

ExAmple 3.3. If $A$ is odd-dimensional and the center of $\operatorname{End}^{0}(A)$ is a CM-field $K$, then $H$ is not semisimple. To show this, note that $A$ is isogenous to a power of a simple odd-dimensional abelian variety $B$ such that $K$ is the center of $\operatorname{End}^{0}(B)$. Then the Hodge groups of $A$ and of $B$ coincide, so we may reduce to the case where $A$ is simple. Let $d=\operatorname{dim}(A)$ and use the notation of the proof of Lemma 2.2. Then $n_{\sigma}+n_{\bar{\sigma}}=2 d /[K: \mathbf{Q}]$. If $H$ were semisimple, then by Theorem $3.1,(A, K$, id) would be of Weil type. We would therefore have $n_{\sigma}=n_{\bar{\sigma}}$, and so $2 d /[K: \mathbf{Q}]$ would be even. However, $d$ is odd and $[K: \mathbf{Q}]$ is even, so this cannot happen.

\section{§ 4. Abelian varieties having purely multiplicative reduction}

Theorem 4.1. Suppose $A$ is an abelian variety over a subfield $F$ of $\mathbf{C}, v$ is a discrete valuation on $F$, and $A$ has purely multiplicative reduction at $v$. Then the Hodge group $H$ of $A$ is semisimple. 
Proof. Since $H$ is semisimple if the Hodge groups of each of its $F$-simple components are, we may reduce to the case where $A$ is an $F$-simple abelian variety with purely multiplicative reduction at $v$. Since the properties of having semisimple Hodge group and having purely multiplicative reduction are invariant under finite extensions of the ground field, we may assume $\operatorname{End}_{F}^{0}(A)=\operatorname{End}^{0}(A)$. Suppose that $H$ is not semisimple. By Theorem 3.1, the center of $\operatorname{End}^{0}(A)$ is a CM-field $K$ such that $(A, K$,id) is not of Weil type, with id the identity embedding of $K$ in $\operatorname{End}^{0}(A)$. Let $L$ be a fixed algebraic closure of the completion $F_{v}$ of $F$ at $v$. Since $A$ has purely multiplicative reduction at $v$, $A$ admits a non-archimedean uniformation; i.e., (see [12] and [14]) there are a discrete subgroup $\Gamma$ of $\mathbf{G}_{m}^{d}\left(F_{v}\right)=\left(F_{v}^{\times}\right)^{d}$, isomorphic to $\mathbf{Z}^{d}$, and a $\operatorname{Gal}\left(L / F_{v}\right)$-equivariant $v$-adically continuous isomorphism $\left(L^{\times}\right)^{d} / \Gamma \cong A(L)$ which for some finite extension $M$ of $F_{v}$ induces an isomorphism $\left(M^{\times}\right)^{d} / \Gamma \cong A(M)$ as $M$-Lie groups. Let $\mathcal{O}$ be the center of $\operatorname{End}(A)$. Then $\mathcal{O}$ is an order in $K$. By Satz 6 of [3], there is a homomorphism $\mathcal{O} \hookrightarrow \operatorname{End}\left(\mathbf{G}_{m}^{d}\right)$ which induces the inclusion $\mathcal{O} \subseteq \operatorname{End}(A)$. Composing with the natural homomorphism

$$
\operatorname{End}\left(\mathbf{G}_{m}^{d}\right) \hookrightarrow \operatorname{End}\left(\operatorname{Hom}\left(\mathbf{G}_{m}, \mathbf{G}_{m}^{d}\right)\right)
$$

and tensoring with $\mathbf{Q}$, we have

$$
K \hookrightarrow \operatorname{End}\left(\operatorname{Hom}\left(\mathbf{G}_{m}, \mathbf{G}_{m}^{d}\right) \otimes \mathbf{Q}\right) .
$$

Therefore, the inclusion of $K$ in $\operatorname{End}^{0}(A)$ induces a $K$-vector space structure on $\operatorname{Hom}\left(\mathbf{G}_{m}, \mathbf{G}_{m}^{d}\right) \otimes \mathbf{Q}$. Tensoring with $M$ makes $\operatorname{Hom}\left(\mathbf{G}_{m}, \mathbf{G}_{m}^{d}\right) \otimes \mathbf{z} M\left(=M^{d}\right)$ into a free $(K \otimes \mathbf{Q} M)$-module. We can view $\left(M^{\times}\right)^{d}$ as a (non-archimedean) analytic variety over $M$. The tangent space to $\left(M^{\times}\right)^{d}$ at 1 is isomorphic to $M^{d}$. By [9] (see also Chapter 2 of [7]), $\left(L^{\times}\right)^{d} / \Gamma$ can be embedded, via theta functions, as an analytic subvariety of a projective space $\mathbf{P}^{n}(L)$, so that the image of $\left(M^{\times}\right)^{d} / \Gamma$ is $A(M)$. Let $T$ denote the analytic tangent space at the origin of the analytic variety $A(M)$. The tangent map is an isomorphism $M^{d} \cong T$. The algebraic tangent space at the origin to the algebraic variety $A$ over $M$ is $\operatorname{Lie}_{M}(A)=\operatorname{Lie}_{F}(A) \otimes_{F} M$, and there is a canonical isomorphism between the analytic and algebraic tangent spaces to $A(M)$ (see subsection 3 of $\S 2$ of Chapter II of [17] or Appendix III of [26]). Therefore, the identity embedding of $K$ into $\operatorname{End}^{0}(A)$ makes $\operatorname{Lie}_{M}(A)$ into a free $\left(K \otimes_{\mathbf{Q}} M\right)$-module. By Lemma $2.2,(A, K$, id $)$ is of Weil type, contradicting our assumptions.

Theorem 4.1 remains true if we replace the assumption that $v$ is a discrete valuation by the assumption that $v$ is a valuation of rank 1 and $A$ admits non-archimedean uniformization (Gerritzen's theorem remains true under these assumptions).

\section{$\S 5$. Bounds on torsion of abelian varieties which do not have purely multiplicative reduction}

It is easy to find uniform bounds on orders of torsion points over number fields for abelian varieties with potential good reduction, or for elliptic curves which do not have multiplicative reduction, at a given discrete valuation (see [20], [19], [2]). In this section we extend these results by finding bounds on torsion subgroups of abelian varieties which do not have purely multiplicative reduction at a given discrete valuation.

Suppose $A$ is a $d$-dimensional abelian variety over a field $F, v$ is a discrete valuation on $F$ with finite residue field $k$ of order $q, n$ is a positive integer relatively prime to $q$, 
and $J$ is a non-zero subgroup of the group $A_{n}(F)$ of points in $A(F)$ of order dividing $n$. Let $A_{v}^{0}$ denote the connected component of the identity of the special fiber $A_{v}$ of the Néron minimal model of $A$ at $v$. Let $a, u$, and $t$ denote respectively the abelian, unipotent, and toric ranks of $A_{v}^{0}$. Then $d=a+t+u$.

If $\lambda$ is a polarization on $A$ defined over an extension of $F$ which is unramified over $v$, define a skew-symmetric Galois-equivariant pairing $e_{\lambda, n}$ on $A_{n}$ by $e_{\lambda, n}(x, y)=$ $e_{n}(x, \lambda(y))$, where $e_{n}$ is the Weil pairing. If $J$ is not isotropic with respect to $e_{\lambda, n}$, and $n$ is a prime number, then $\zeta_{n} \in F$, so the prime $n$ can be bounded independent of $A$ (with a bound depending on $F$ ). Therefore, the more interesting case is when $J$ is an isotropic subgroup of $A_{n}(F)$. If $J$ is a maximal isotropic subgroup of $A_{n}(F)$, and $A$ does not have semistable reduction at $v$, then $n \leq 4$, by Theorem 6.2 of [21]. The remaining case to consider is the case where $A$ has semistable reduction at $v$. Theorem 5.3 below implies that in this case we can bound $n$ in terms of $q$ and $d$, as long as $A$ does not have purely multiplicative reduction at $v$. Note that if $P$ is a point of $A(F)$ of order $n$ which reduces to a point of $A_{v}^{0}$, then $n$ is bounded above by $\# A_{v}^{0}(k)$. Therefore even in the case of abelian varieties with purely multiplicative reduction one can easily bound, by a constant depending only on $d$ and $q$, the orders of torsion points whose reductions lie in $A_{v}^{0}$. As was the case for elliptic curves, the most difficult case is the case when the reduction is purely multiplicative and the reductions of the torsion points do not lie in the identity connected component of the special fiber of the Néron minimal model.

Lemma 5.1 (Lemma 1 on pp. 494-495 of [16]). If $A$ is an abelian variety over a field $F, v$ is a discrete valuation on $F$, and $n$ is a positive integer relatively prime to the residue characteristic of $v$, then $\left(A_{v}^{0}\right)_{n}$ is a free $\mathbf{Z} / n \mathbf{Z}$-module of rank $2 a+t$.

Proposition 5.2. Suppose $A$ is an abelian variety over a field $F, v$ is a discrete valuation on $F$ with finite residue field $k$ of order $q, n$ is a positive integer relatively prime to $q$, and $J$ is a subgroup of $A_{n}(F)$. Suppose there is a positive constant $\epsilon$ such that $|J| \geq n^{t+2 u+\epsilon}$. Then $n \leq\left(\#\left(A_{v}^{0}\right)_{n}(k)\right)^{1 / \epsilon} \leq\left(\# A_{v}^{0}(k)\right)^{1 / \epsilon}$.

Proof. Let $d=\operatorname{dim}(A)$. Via the reduction map we may view $A_{n}(F)$, and therefore $J$, as a subgroup of $\left(A_{v}\right)_{n}$ (see [16]). Therefore, \#J\#( $\left.A_{v}^{0}\right)_{n}$ divides $n^{2 d} \#(J \cap$ $\left.\left(A_{v}^{0}\right)_{n}\right)$. Thus by Lemma $5.1, \# J$ divides $n^{t+2 u} \#\left(J \cap\left(A_{v}^{0}\right)_{n}\right)$. Therefore,

$$
n^{\epsilon} \leq \#\left(J \cap\left(A_{v}^{0}\right)_{n}\right) \leq \#\left(A_{v}^{0}\right)_{n}(k) \leq \# A_{v}^{0}(k) .
$$

TheOrem 5.3. Suppose $A$ is a d-dimensional abelian variety over a field $F, v$ is a discrete valuation on $F$ with finite residue field $k$ of order $q, n$ is a positive integer relatively prime to $q$, and $A_{n}(F)$ has a subgroup of order $n^{d}$. Suppose the reduction of $A$ at $v$ is semistable but not purely multiplicative. Then

$$
n \leq(1+\sqrt{q})^{2 a}(1+q)^{t} \leq(1+\sqrt{q})^{2 d} .
$$

Proof. Since $A$ has semistable reduction at $v, u=0$. Since the reduction of $A$ at $v$ is not purely multiplicative, $a \geq 1$. Applying Proposition 5.2 with $\epsilon=1$, we have $n \leq \# A_{v}^{0}(k)$. Since $A$ has semistable reduction at $v, A_{v}^{0}$ is an extension of an abelian variety $B$ by a torus $T$. We have the Weil bound $\# B(k) \leq(1+\sqrt{q})^{2 a}$. Similarly, we have the bound $\# T(k) \leq(1+q)^{t}$, as follows. Let $X$ be the group of characters of $T \otimes \bar{k}$. The Frobenius element of $\operatorname{Gal}(\bar{k} / k)$ acts on $X$, say by $\varphi_{0}$. Since the torus $T$ 
splits over some finite extension of $k, \operatorname{Gal}(\bar{k} / k)$ acts on $X$ through a finite quotient, so all the eigenvalues of $\varphi_{0}$ have absolute value 1 . Therefore all eigenvalues of $q-\varphi_{0}$ are non-zero and have absolute value at most $1+q$. We have (see Theorem 6.2 in $\S 1$ of Chapter VI of [23])

$$
\# T(k)=\left|\operatorname{det}\left(q-\varphi_{0}\right)\right| \leq(1+q)^{t} .
$$

Therefore,

$$
\# A_{v}^{0}(k) \leq(1+\sqrt{q})^{2 a}(1+q)^{t}=(1+\sqrt{q})^{2 a}(1+q)^{d-a} \leq(1+\sqrt{q})^{2 d} .
$$

If $c$ and $d$ are positive integers, let $f(c, d)$ be the maximum of the orders of the elements of $\mathrm{GL}_{2 d}(\mathbf{Z} / c \mathbf{Z})$.

THEOREM 5.4. Suppose $A$ is a d-dimensional abelian variety over a number field $F$ of degree $m, v$ is a discrete valuation on $F$ at which $A$ does not have purely multiplicative reduction, $p$ is the residue characteristic of $v, n$ and $r$ are positive integers not divisible by $p, r \geq 3$, and $A_{n}(F)$ has a subgroup of order $n^{d}$. Then

$$
n \leq\left(1+p^{m f(r, d) / 2}\right)^{2 d} .
$$

Proof. By a theorem of Raynaud (Proposition 4.7 of [4]), $A$ has semistable reduction at the discrete valuations on the field $F\left(A_{r}\right)$ of residue characteristic not dividing $r$. Let $v^{\prime}$ be a valuation on $F\left(A_{r}\right)$ extending $v$, and let $k$ be the corresponding residue field. Then \#k divides $p^{m f}$, where $f$ is the order of Frobenius at $v^{\prime}$ in $\operatorname{Gal}\left(F\left(A_{r}\right) / F\right)$. Since $\operatorname{Gal}\left(F\left(A_{r}\right) / F\right)$ injects into $\mathrm{GL}_{2 d}(\mathbf{Z} / r \mathbf{Z})$, \#k divides $p^{m f(r, d)}$. The result now follows from Theorem 5.3.

Corollary 5.5. Suppose $A$ is a d-dimensional abelian variety over a number field $F$ of degree $m$, and suppose the Hodge group of $A$ is not semisimple. Suppose $n$ is a positive integer and $A_{n}(F)$ has a subgroup of order $n^{d}$. Then

$$
n \leq\left[\left(1+2^{m f(3, d) / 2}\right)\left(1+3^{m f(4, d) / 2}\right)\right]^{2 d}<\left(1+10^{-11}\right) \cdot\left(2^{3^{4 d^{2}}} \cdot 3^{4^{4 d^{2}}}\right)^{m d} .
$$

Proof. The result follows from Theorem 4.1, by applying Theorem 5.4 with $p=2$, $r=3$ to bound the prime-to-two part of $n$, and with $p=3, r=4$ to bound the prime-to-three part of $n$. The final inequality follows from the bound $f(c, d) \leq$ \# $\mathrm{GL}_{2 d}(\mathbf{Z} / c \mathbf{Z})<c^{4 d^{2}}$.

The bounds on $n$ given in Corollary 5.5 were shown in Theorem 3.3 and Remark 2 of [20] to be bounds on the orders of torsion subgroups of abelian varieties with potential good reduction at discrete valuations of residue characteristics 2 and 3 .

If we assume the existence of a polarization on $A$ of degree prime to $n$ (for example, a principal polarization) we obtain stronger bounds. The following results give bounds on torsion subgroups of order prime to the degree of a given polarization.

TheOREM 5.6. Suppose A is a d-dimensional abelian variety over a number field $F$ of degree $m, v$ is a discrete valuation on $F$ at which $A$ does not have purely multiplicative reduction, $p$ is the residue characteristic of $v, \ell$ is a prime number, $\ell \neq p, \quad J$ is a subgroup of $A_{\ell}(F)$ of order $\ell^{d}, \lambda$ is a polarization on $A$ defined over an extension of $F$ unramified at $v$, and $\ell$ does not divide the degree of $\lambda$. Then

$$
\ell \leq\left(1+p^{m / 2}\right)^{2 d} \text {. }
$$


ProOF. Since $\ell$ does not divide the degree of $\lambda$, the pairing $e_{\lambda, \ell}$ is nondegenerate. If $J$ is not isotropic with respect to $e_{\lambda, \ell}$, then $F$ contains a primitive $\ell$-th root of unity. Therefore $\ell-1$ divides $[F: \mathbf{Q}]$, so $\ell \leq 1+m$.

Suppose $J$ is isotropic with respect to $e_{\lambda, \ell}$. Then $J$ is a maximal isotropic subgroup of $A_{\ell}$ (since $\# J=\ell^{d}$ and $e_{\lambda, \ell}$ is nondegenerate). If $A$ does not have semistable reduction at $v$, then $\ell \leq 3$ by Theorem 6.2 of [21]. If $A$ has semistable reduction at $v$, then $\ell \leq\left(1+p^{m / 2}\right)^{2 d}$ by Theorem 5.3. The result now follows since $\left(1+p^{m / 2}\right)^{2 d}$ is greater than 3 and than $1+m$.

COROLlary 5.7. Suppose $(A, \lambda)$ is a d-dimensional polarized abelian variety over a number field $F$ of degree $m$, and suppose the Hodge group of $A$ is not semisimple. Suppose $\ell$ is a prime number which does not divide the degree of $\lambda$, and $J$ is a subgroup of $A_{\ell}(F)$ of order $\ell^{d}$. Then

$$
\ell \leq\left(1+2^{m / 2}\right)^{2 d}
$$

Proof. By Theorem 4.1, $A$ does not have purely multiplicative reduction at any discrete valuations. Since $2<\left(1+2^{m / 2}\right)^{2 d}$, we may assume $\ell$ is an odd prime, and we obtain the result by applying Theorem 5.6 with $p=2$.

The proof of Theorem 5.6 shows that $\ell \leq \max \left\{1+m,(1+\sqrt{q})^{2 d}\right\}$, where $q$ is the order of the residue field of $v$. Therefore in Corollary 5.7 we can conclude that $\ell \leq \max \left\{1+m,(1+\sqrt{f})^{2 d}\right\}$, where $f$ is the minimal order of the residue fields of the valuations on $F$ of residue characteristic 2 .

Corollary 5.8. Suppose $(A, \lambda)$ is a d-dimensional polarized abelian variety over a number field $F$ of degree $m$, and suppose the Hodge group of $A$ is not semisimple. Suppose $n$ is a positive integer relatively prime to the degree of $\lambda$, and $J$ is a subgroup of $A_{n}(F)$ of order $n^{d}$ which is a maximal isotropic subgroup with respect to $e_{\lambda, n}$. Then

$$
n \leq\left(1+2^{m / 2}\right)^{2 d}\left(1+3^{m / 2}\right)^{2 d} .
$$

Proof. By Theorem 4.1, $A$ does not have purely multiplicative reduction at any discrete valuations. The prime-to- $p$ part of $n$ is bounded above by 4 if there is a valuation on $F$ of residue characteristic $p$ at which $A$ does not have semistable reduction (by Theorem 6.2 of [21]), and otherwise is bounded above by $\left(1+p^{m / 2}\right)^{2 d}$ (by Theorem 5.3). Note that $4<\left(1+p^{m / 2}\right)^{2 d}$. The result follows by letting $p=2,3$.

\section{Список литературы}

1. Faltings G. Endlichkeitssätze für abelsche Varietäten über Zahlkörpern // Invent. Math. 1983. V. 73. P. 349-366.

2. Flexor M., Oesterlé J. Sur les points de torsion des courbes elliptiques // Astérisque. 1990. V. 183. P. 25-36.

3. Gerritzen L. Über Endomorphismen nichtarchimedischer holomorpher Tori // Invent. math. 1970. V. 11. P. 27-36.

4. Grothendieck A. Modèles de Néron et monodromie // Groupes de monodromie en géometrie algébrique, SGA7 I / ed. A. Grothendieck. Lecture Notes in Math.. V. 288. Berlin-Heidelberg-New York: Springer, 1972. P. 313-523.

5. Hazama F. Algebraic cycles on nonsimple abelian varieties // Duke Math. J. 1989. V. 58. P. 31-37.

6. Humphreys J. E. Linear Algebraic Groups, Graduate Texts in Mathematics. V. 21. New York-Heidelberg-Berlin: Springer-Verlag, 1975. 
7. Manin Yu. I. p-adic automorphic functions // J. Soviet Math. 1976. V. 5. №1. P. 279-333; // Itogi Nauki i Tekhniki. Sovremennye Problemy Matematiki. V. 3, 1974. P. 5-92.

8. Moonen B., Zarhin Yu. G. Hodge classes and Tate classes on simple abelian fourfolds // Duke Math. J. 1995. V. 77. P. 553-581.

9. Morikawa $H$. Theta functions and abelian varieties over valuation fields of rank one I. // Nagoya Math. J. 1962. V. 20. P. 1-27.

10. Mumford D. Families of Abelian Varieties // Algebraic Groups and Discontinuous Subgroups, Proc. Sympos. Pure Math.. V. 9. Providence, R. I.: Amer. Math. Soc., 1966. P. 347-351.

11. Mumford D. A note of Shimura's paper "Discontinuous groups and abelian varieties" // Math. Ann. 1969. V. 181. P. 345-351.

12. Mumford $D$. An analytic construction of degenerating abelian varieties over complete rings // Comp. math. 1972. V. 24. P. 239-272.

13. Mustafin G. A. Families of algebraic varieties and invariant cycles // Math. USSR Izvestija 1986. V. 27. № 2. P. 251-278; // Izv. Akad. Nauk SSSR Ser. Mat. 1985. V. 49. № 5. P. 948-978, 1119.

14. Raynaud $M$. Variétés abéliennes et géométrie rigide // Actes, Congrès intern. math., Nice. V. 1, 1970. P. 473-477.

15. Ribet K. Hodge classes on certain types of abelian varieties // Amer. J. Math. 1983. V. 105. P. 523-538.

16. Serre J-P., Tate J. Good reduction of abelian varieties // Ann. of Math. 1968. V. 88. P. $492-517$.

17. Shafarevich I. R. Basic Algebraic Geometry. Berlin-Heidelberg-New York: Springer-Verlag, 1974; Osnovy algebraicheskoi geometrii. Moscow: Nauka, 1972.

18. Shimura $G$. On analytic families of polarized abelian varieties and automorphic functions // Ann. of Math. 1963. V. 78. P. 149-192.

19. Silverberg A. Torsion points on abelian varieties of CM-type // Comp. math. 1988. V. 68. P. 241-249.

20. Silverberg A. Points of finite order on abelian varieties // $p$-adic Methods in Number Theory and Algebraic Geometry / ed. A. C. Adolphson, S. Sperber, M. D. Tretkoff,. Contemp. math.. V. 133. Providence: Amer. Math. Soc., 1992. P. 175-193.

21. Silverberg A., Zarhin Yu. G. Semistable reduction and torsion subgroups of abelian varieties // Ann. Inst. Fourier. 1995. V. 45. № 2. P. 403-420.

22. Tankeev S. G. On algebraic cycles of abelian varieties II // Math. USSR Izvestija. 1980. V. 14. № 2. P. 383-394; // Izv. Akad. Nauk SSSR Ser. Mat. 1979. V. 43. P. 418-429.

23. Voskresensky V. E. Algebraic Tori. Moscow: Nauka, 1977.

24. Zarhin Yu. G. Torsion of abelian varieties in finite characteristic // Math. Notes. 1978. V. 22. № 1. P. 493-498; // Mat. Zametki. 1977. V. 22. № 1. P. 3-11.

25. Zarhin $Y u$. G. Abelian varieties having a reduction of K3 type // Duke Math. J. 1992. V. 65. P. 511-527.

26. Weil A. Foundations of Algebraic Geometry, AMS Colloquium Publications. Revised Edition. V. 29. Providence, R. I.: American Mathematical Society, 1962.

Mathematics Department,

Ohio State University,

Columbus, OH 43210-1174, USA

Поступило в редакцию

E-mail address: silver@math.ohio-state.edu

Mathematics Department,

Pennsylvania State University,

University Park, PA 16802, USA

and

Institute for Mathematical Problems in Biology,

Russian Academy of Sciences,

Pushchino, Moscow Region, 142292, RUSSIA

E-mail address: zarhin@math.psu.edu 